\title{
QED radiative corrections for Polarized Lepton-Proton Scattering
}

\author{
R.-D. Bucoveanu* \\ PRISMA+ Cluster of Excellence, Institut für Kernphysik, Johannes Gutenberg-Universität, \\ D-55099 Mainz, Germany \\ E-mail: rabucoveduni-mainz.de

\section{H. Spiesberger} \\ PRISMA+ Cluster of Excellence, Institut für Physik, Johannes Gutenberg-Universität, D-55099 \\ Mainz, Germany \\ E-mail: spiesbereuni-mainz.de
}

We discuss QED radiative corrections at first and second order for the parity violating asymmetry $A_{\mathrm{PV}}$ in elastic electron proton scattering. A measurement of $A_{\mathrm{PV}}$ is planned at low energies and with high precision by the forthcoming P2 experiment at the new MESA facility in Mainz. Bremsstrahlung leads to a shift of the momentum transfer $Q^{2}$ which affects the determination of the weak charge of the proton.

23rd International Spin Physics Symposium - SPIN2018 -

10-14 September, 2018

Ferrara, Italy

${ }^{*}$ Speaker. 


\section{Introduction}

The upcoming P2 experiment at the MESA facility in Mainz plans to measure the weak mixing angle with a precision of $0.15 \%$ [1] by determining the weak charge of the proton from a measurement of the parity violating asymmetry $A_{P V}$ of polarized electrons scattered off a proton target. The parity violating asymmetry is proportional to the momentum transfer $Q^{2}$ and is, therefore, affected by QED radiative corrections which lead to a shift of $Q^{2}$. In this work we discuss these radiative corrections at first and second order. A previous calculation of first order QED corrections to the parity violating asymmetry, can be found in $[2,3]$.

\section{First-order QED corrections}

The leading order asymmetry between cross sections for incident electrons with positive and negative helicities, $\sigma_{ \pm}^{(0)}$, at low momentum transfer, is given by

$$
A_{P V}^{(0)}=\frac{\sigma_{+}^{(0)}-\sigma_{-}^{(0)}}{\sigma_{+}^{(0)}+\sigma_{-}^{(0)}}=-\frac{G_{F} Q^{2}}{4 \sqrt{2} \pi \alpha}\left[Q_{W}^{p}-F\left(Q^{2}\right)\right],
$$

where $Q_{W}^{p}$ is the weak charge of the proton and $F\left(Q^{2}\right)$ comprises form factors describing the proton structure. $F\left(Q^{2}\right)$ is small at the $Q^{2}$ values relevant for the P2 experiment. Uncertainties due to $F\left(Q^{2}\right)$ will be known with sufficiently high precision from a combination of previous measurements and ancillary measurements at backward scattering angles planned at the P2 experiment. In Eq. (2.1) we see that the parity violating asymmetry is proportional to $Q^{2}$. Even though the QED corrections are parity conserving and do not affect the weak charge of the proton directly, they lead to a shift of the momentum transfer. If an experimental event-by-event determination of $Q^{2}$ is not possible, QED corrections have to be applied in the analysis to extract $Q_{W}^{p}$ from the measured asymmetry. Therefore, these corrections have to be calculated from theory with high precision.

From the measurement of the scattering angle one can only obtain information about what we can call leptonic momentum transfer (see [4]) defined as

$$
Q_{l}^{2}=-\left(l-l^{\prime}\right)^{2}
$$

where $l$ and $l^{\prime}$ are the 4-momenta of the initial and final electrons. However, taking into account bremsstrahlung effects (see Fig. 1), a photon with momentum $k$ will shift the momentum transfer to the true value given by

$$
Q^{2}=-\left(l-l^{\prime}-k\right)^{2} .
$$
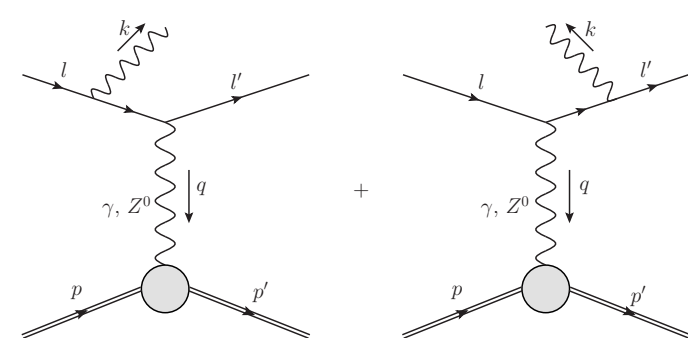

Figure 1: Feynman diagrams describing first-order bremsstrahlung. 


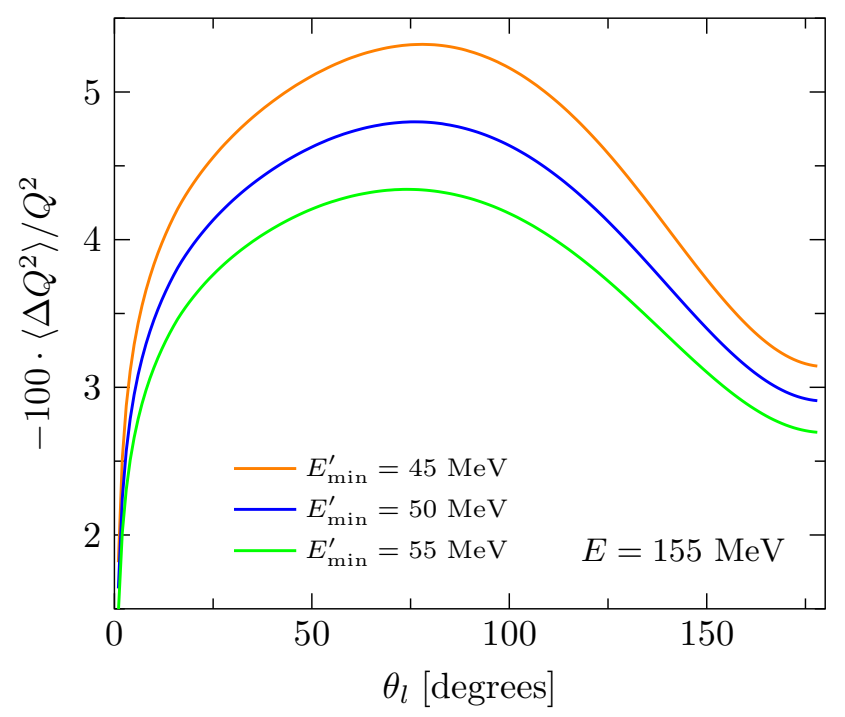

Figure 2: The average relative shift of the momentum transfer due to hard photon radiation as a function of the electron scattering angle and for different minimum cuts for the energy of the observed scattered electron. The beam energy is $E=155 \mathrm{MeV}$ as expected for the Mainz P2 experiment.

We define an average shift of the momentum transfer by

$$
\left\langle\Delta Q^{2}\right\rangle=\frac{1}{\sigma^{(0+1)}} \int \mathrm{d}^{4} \sigma_{1 \gamma}^{(1)} \Delta Q^{2},
$$

where $\sigma^{(0+1)}$ is the total cross section including $\mathscr{O}(\alpha)$ corrections, while $\mathrm{d}^{4} \sigma_{1 \gamma}^{(1)}$ is the differential cross section for the process with one additional photon radiated into the final state. For a more detailed description of these cross sections see Ref. [4] and the discussion below. $\Delta Q^{2}=Q^{2}-Q_{l}^{2}$ is defined as the difference between the true $Q^{2}$ and the leptonic $Q_{l}^{2}$. The integration in this expression can be performed numerically over the entire phase-space, since the product $\mathrm{d}^{4} \sigma_{1 \gamma}^{(1)} \Delta Q^{2}$ is infrared (IR) finite. Results for the kinematical conditions of the Mainz P2 experiment are shown in Fig. 2. We find that the average shift of the momentum transfer has a strong dependence on kinematic variables. Its dependence on the scattering angle is seen in Fig. 2. We also find a strong dependence on the cut for the minimum value of the scattered electron's energy. This cut is related to the detector acceptance of the experiment.

While a determination of the true momentum transfer is difficult or impossible, the dependence of the asymmetry on the electron scattering angle, $\theta_{l}$, is directly accessible in the experiment. We therefore study the effect of QED radiative corrections on the $\theta_{l}$-dependence of $A_{P V}$. The asymmetry including $\mathscr{O}(\alpha)$ QED corrections is defined as

$$
A_{P V}^{(0+1)}=\frac{\sigma_{+}^{(0+1)}-\sigma_{-}^{(0+1)}}{\sigma_{+}^{(0+1)}+\sigma_{-}^{(0+1)}}=\frac{\sigma_{P}^{(0)}+\sigma_{P}^{(1)}}{\sigma^{(0)}+\sigma^{(1)}},
$$

where the upper index $(n), n=0,1$, or $0+1$, denotes the order at which the cross section is evaluated, relative to the leading order. The polarization-dependent part of the cross section is labelled with a lower index $P$, i.e. $\sigma_{P}^{(n)}=\sigma_{+}^{(n)}-\sigma_{-}^{(n)}$. The first order unpolarized cross section is 
given by (see [4])

$$
\sigma^{(1)}=\sigma_{\text {non-rad }}^{(1)}+\sigma_{1 h \gamma}^{(1)}
$$

where the non-radiative cross section, given by

$$
\sigma_{\text {non-rad }}^{(1)}=\int \mathrm{d} \sigma^{(0)}\left[\delta_{1-\text { loop }}^{(1)}+\delta_{1 s \gamma}^{(1)}(\Delta)\right],
$$

is IR finite and contains both 1-loop corrections and corrections due to one radiated soft photon. In this expression we have used correction factors relative to the Born-level cross section $\sigma^{(0)}$. The soft-photon part can be calculated analytically, integrating over the photon energy up to the cut-off $\Delta$, by using a soft-photon approximation as described in [4]. The cross section with a hard photon in the final state, i.e. with photon energies above the cut-off $\Delta$, is infrared finite and the phase space integration can be performed numerically. For one hard photon at tree level, we can write

$$
\sigma_{1 h \gamma}^{(1)}=\int_{E_{\gamma}>\Delta} \mathrm{d}^{4} \sigma_{1 \gamma}^{(1)}
$$

The sum of non-radiative and hard-photon contributions has to be independent of $\Delta$. However, when we use the soft-photon approximation to calculate the non-radiative contributions and due to numerical uncertainties we don't expect the result to be exactly $\Delta$-independent. We show the $\Delta$-dependence of the correction factor in Fig. 3. We find that the soft-photon approximation starts to distort the result at large values of $\Delta$. At very small $\Delta$, the numerical cancellation of $\Delta$-dependent terms lead to fluctuations and increasing numerical uncertainties. The fact that there is a wide plateau for which the numerical result for the cross section is independent of $\Delta$ proves that our approach leads to reliable predictions. Furthemore, we have checked that our calculation of the first order cross-section for scattering of unpolarized electrons agrees with [5] and [6].

In a similar way the polarized cross section can be separated into a non-radiative and a radiative part (see [7]) as

$$
\sigma_{P}^{(1)}=\sigma_{\text {non-rad }, P}^{(1)}+\sigma_{1 h \gamma, P}^{(1)},
$$

where the non-radiative part is given, as for the unpolarized cross section, by the sum of QED 1-loop and one soft photon bremsstrahlung corrections. QED 1-loop corrections for one-photon exchange are the same as for the unpolarized cross section. The QED 1-loop corrections for $Z_{0}$ exchange are shown in Fig 4. With on-shell renormalization, the self-energy diagrams vanish and the only diagram contributing in this case is the photonic vertex correction.

Finally the hard-photon polarized cross section is defined as

$$
\sigma_{1 h \gamma, P}^{(1)}=\int_{E_{\gamma}>\Delta} \mathrm{d}^{4} \sigma_{1 \gamma, P}^{(1)}=\int_{E_{\gamma}>\Delta}\left(\mathrm{d}^{4} \sigma_{1 \gamma,+}^{(1)}-\mathrm{d}^{4} \sigma_{1 \gamma,-}^{(1)}\right) .
$$

This contirubution to the cross section is IR finite and the integration can be performed numerically as in the case of the unpolarized cross section. The total asymmetry including $\mathscr{O}(\alpha)$ corrections is therefore also independent of the cut-off $\Delta$ and the difference between the leading order asymmetry and the asymmetry with $\mathscr{O}(\alpha)$ corrections is found to be of the same size as the relative shift of $Q^{2}$. This is demonstrated by the numerical results shown in Fig. 5. 


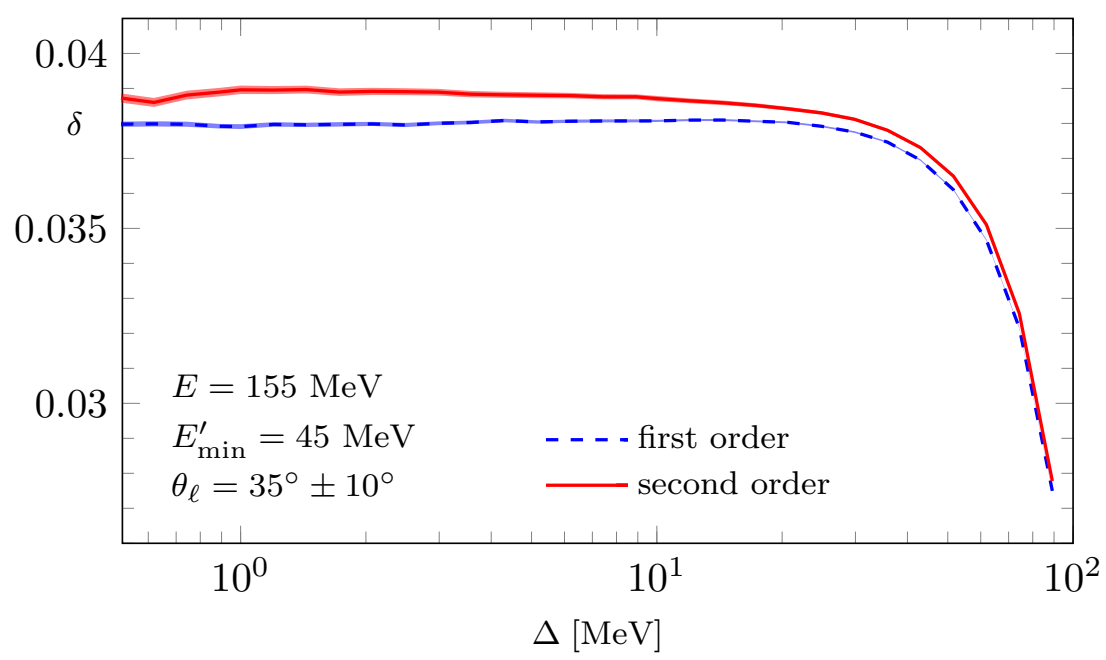

Figure 3: Test of the $\Delta$-independence of the complete correction factors when non-radiative contributions and hard-photon radiative effects are added, at first order $\delta=\sigma^{(0+1)} / \sigma^{(0)}-1$ and at second order $\delta=$ $\sigma^{(0+1+2)} / \sigma^{(0)}-1$. Beam energy $(E=155 \mathrm{MeV})$, the range of the scattering angle $\left(25^{\circ} \leq \theta_{l} \leq 45^{\circ}\right)$, and the minimum energy of the scattered electron $\left(E_{\min }^{\prime}=45 \mathrm{MeV}\right)$ is chosen for electron scattering at the Mainz P2 experiment.
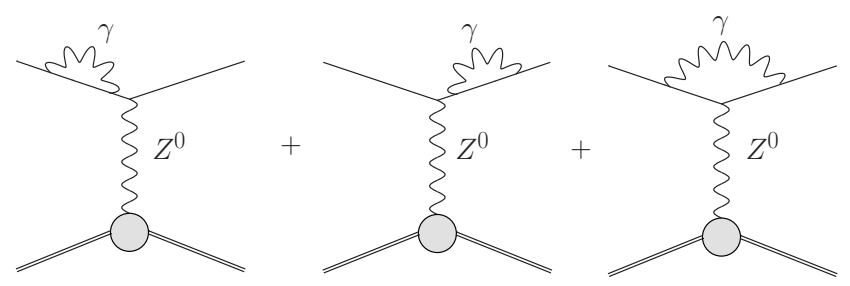

Figure 4: One-loop photonic corrections to $Z_{0}$ exchange.

\section{Second-order QED corrections}

Since the first-order QED corrections lead to a large effect on the measured value of the parity violating asymmetry, it is important to include also $\mathscr{O}\left(\alpha^{2}\right)$ QED corrections, or to make sure they have a negligible effect.

The parity violating asymmetry including $\mathscr{O}\left(\alpha^{2}\right)$ corrections is written as

$$
A_{P V}^{(0+1+2)}=\frac{\sigma_{+}^{(0+1+2)}-\sigma_{-}^{(0+1+2)}}{\sigma_{+}^{(0+1+2)}+\sigma_{-}^{(0+1+2)}}=\frac{\sigma_{P}^{(0)}+\sigma_{P}^{(1)}+\sigma_{P}^{(2)}}{\sigma^{(0)}+\sigma^{(1)}+\sigma^{(2)}},
$$

where $\sigma^{(2)}$ is the $\mathscr{O}\left(\alpha^{2}\right)$ correction to the unpolarized cross section while $\sigma_{P}^{(2)}$ is the difference between the $\mathscr{O}\left(\alpha^{2}\right)$ corrections of the cross sections for positive and negative helicities of the initial electron. Following [4], the unpolarized cross with second order corrections is written as

$$
\sigma^{(2)}=\sigma_{\text {non-rad }}^{(2)}+\sigma_{1 h \gamma}^{(2)}+\sigma_{2 h \gamma}^{(2)}
$$

where

$$
\sigma_{\text {non-rad }}^{(2)}=\sigma_{2-\text { loop }}^{(2)}+\sigma_{1-\mathrm{loop}+1 s \gamma}^{(2)}+\sigma_{2 s \gamma}^{(2)}, \quad \sigma_{1 h \gamma}^{(2)}=\sigma_{1-\mathrm{loop}+1 h \gamma}^{(2)}+\sigma_{1 s \gamma+1 h \gamma}^{(2)},
$$


and the labels indicate whether the different contributions are due to 1- or 2-loop diagrams or from 1- or 2- soft or hard photon radiation. The non-radiative part is rendered IR-finite by including loop diagrams: $\sigma_{\text {non-rad }}^{(2)}$ contains two-loop contributions and mixed soft-photon + one-loop parts, while $\sigma_{1 h \gamma}^{(2)}$ contains one-loop corrections to the radiative process with one hard photon. An analytical expression for the 2-loop corrections can be found for example in [8]. Using relative correction factors, the non-radiative part can be written as

$$
\sigma_{\text {non-rad }}^{(2)}=\int \mathrm{d} \sigma^{(0)}\left[\delta_{2-\text { loop }}^{(2)}+\delta_{1-\text { loop }+1 s \gamma}^{(2)}(\Delta)+\delta_{2 s \gamma}^{(2)}(\Delta)\right] .
$$

Finally, the cross section for two hard photons is given by

$$
\sigma_{2 h \gamma}^{(2)}=\int_{E_{\gamma}, E_{\gamma}^{\prime}>\Delta} \mathrm{d}^{7} \sigma_{2 \gamma}^{(2)} .
$$

It is free of IR singularities and can be calculated numerically as described in [4]. The separate parts of Eq. (3.2) depend on the cut-off $\Delta$, but the total result has to be independent of this parameter. Figure 3 shows that the cancellation of $\Delta$-dependent terms works also at second-order in a wide range of values for the IR cut-off $\Delta$.

As in the case of first order corrections, the second order QED corrections for the polarized cross section are defined in the same way as for unpolarized scattering:

$$
\sigma_{P}^{(2)}=\sigma_{\text {non-rad }, P}^{(2)}+\sigma_{1 h \gamma, P}^{(2)}+\sigma_{2 h \gamma, P}^{(2)} .
$$

The definitions of the individual parts follow in the same way as for the unpolarized counter parts (see [7] for more details) and the total polarized cross section is IR finite and independent of the cut-off $\Delta$ on the radiated photons energies. A numerical calculation of the parity-violating asymmetry results in very small second-order corrections. This is shown in Fig. 5 where the curves for $A_{P V}$ at first and at second order are almost indistinguishable. In fact, radiative corrections for the asymmetry are due to the shift of the momentum transfer $Q^{2}$. This kinematical effect is

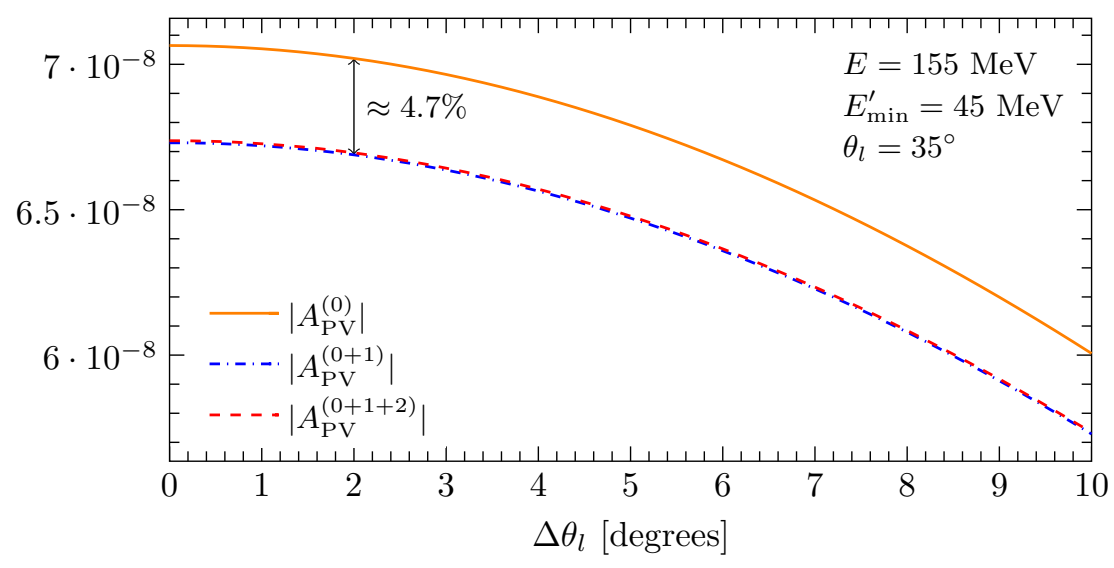

Figure 5: The parity violating asymmetry $A_{P V}$ at leading order and including $\mathscr{O}(\alpha)$ and $\mathscr{O}\left(\alpha^{2}\right)$ QED corrections. Kinematic variables are again chosen as relevant for the Mainz P2 experiment. The asymmetry is shown as a function of the scattering angle acceptance $\Delta \theta_{l}$, i.e. cross sections are integrated over the electron scattering angle in the range $35^{\circ}-\Delta \theta_{l} \leq \theta_{l} \leq 35^{\circ}+\Delta \theta_{l}$. 
already fully present if there is one radiated photon and second-order corrections contribute indeed only at the expected level with an additional factor of $\alpha / \pi$. Second-order QED corrections to the asymmetry have a negligibly small effect.

\section{Conclusions}

In this work we discussed the effects of QED radiative corrections at first and second order to the parity violating asymmetry in elastic electron proton scattering. We found that, at first order, hard photon bremsstrahlung corrections lead to a shift of the momentum transfer $Q^{2}$ of approximately $5 \%$ for P2 kinematics. Since $A_{\mathrm{PV}}$ is proportional to $Q^{2}$ the difference between the leading order asymmetry and the asymmetry with first order QED corrections is also of this order of magnitude. Second-order QED corrections, however, contribute only very little. In addition to the QED corrections described above, also box graph contributions will have to be included in order to control the relation between the observed asymmetry and the weak charge of the proton at the required level of precision. A corresponding full calculation is underway.

\section{Acknowledgments}

This work is supported by the Deutsche Forschungsgemeinschaft (DFG) in the framework of the collaborative research center SFB1044 "The Low-Energy Frontier of the Standard Model: From Quarks and Gluons to Hadrons and Nuclei”.

\section{References}

[1] D. Becker, R. Bucoveanu, C. Grzesik, et al., Eur. Phys. J. A (2018) 54, arXiv:1802.04759 [nucl-ex].

[2] S. Barkanova, A. Aleksejevs, P.G. Blunden, arXiv:nucl-th/0212105.

[3] A. Aleksejevs, S. Barkanova, P. G. Blunden, N. Deg, arXiv:0707.0657 [nucl-th].

[4] R.-D. Bucoveanu and H. Spiesberger, to appear in Eur. Phys. J. A, arXiv:1811.04970 [hep-ph].

[5] A. V. Gramolin, V. S. Fadin, A. L. Feldman, R. E. Gerasimov, D. M. Nikolenko, I. A. Rachek and D. K. Toporkov, J. Phys. G 41 (2014) 115001 [arXiv:1401.2959 [nucl-ex]].

[6] M. Vanderhaeghen, J. M. Friedrich, D. Lhuillier, D. Marchand, L. Van Hoorebeke and J. Van de Wiele, Phys. Rev. C 62 (2000) 025501 [hep-ph/0001100].

[7] R.-D. Bucoveanu and H. Spiesberger, in preparation.

[8] P. Mastrolia and E. Remiddi, Nucl. Phys. B 664 (2003) 341 [hep-ph/0302162]. 